\title{
Effects of a uniform magnetic field on a growing or collapsing bubble in a weakly viscous conducting fluid
}

\author{
K. H. Kang \\ Department of Mechanical Engineering, Pohang University of Science and Technology, San 31, \\ Hyoja-dong, Pohang 790-784, Korea \\ I. S. Kang \\ Department of Chemical Engineering, Pohang University of Science and Technology, San 31, Hyoja-dong, \\ Pohang 790-784, Korea \\ C. M. Lee \\ Department of Mechanical Engineering, Pohang University of Science and Technology, San 31, \\ Hyoja-dong, Pohang 790-784, Korea
}

(Received 13 May 1999; accepted 14 September 2001)

\begin{abstract}
The effects of a uniform magnetic field on a growing or collapsing bubble are investigated. The governing equations for the volume and shape modes of oscillation are derived. To obtain the pressure correction due to the electromagnetic field, the perturbed flow by the electromagnetic force is analyzed with the aid of the domain perturbation method. The viscous effect is assumed to be confined to a thin layer adjacent to the bubble surface. It is shown that the electromagnetic field exerts a damping force on the volume mode so that both the growing and collapsing speeds of a bubble are reduced. The magnetic field also affects the shape mode by contributing to a forcing term. Due to the forcing term, the shape of a growing-collapsing bubble becomes unstable even in the case of no initial disturbance. (C) 2002 American Institute of Physics.
\end{abstract}

[DOI: $10.1063 / 1.1425410]$

\section{INTRODUCTION}

The violent growth or collapse of bubbles, in the form of either cavitation or vapor explosion, has the potential to damage the surrounding objects. ${ }^{1}$ The most common example is the erosion of hydraulic machinery due to hydrodynamic cavitations. In particular, the cavitation in flowing liquid metal is an important subject in the design and safety analysis of the liquid-metal cooled fast breeder reactor. In addition, a catastrophic accident could occur due to the direct fuel-coolant interaction followed by the loss of coolant flow, or a large leak of chemically reactive liquid metal substances, such as sodium. ${ }^{2-4}$ In a certain liquid metal magnetohydrodynamic (MHD) energy conversion system, the circulation of conducting fluids is driven by the volume expansion of gases or water. The direct contact boiling of liquid can be explosive enough to cause damage to the surrounding objects. ${ }^{5}$

It has been anticipated that the magnetic field can be used to control the growth or collapse of bubbles in conducting fluids. Such an idea is based on past experimental observations. Shalobasov and Shal'nev ${ }^{6}$ showed, in a cavitation tunnel filled with tap water, that a magnetic field of less than 1 tesla can significantly change the degree of cavitation erosion. Later, Shal'nev et al. ${ }^{7}$ showed that the growth rate of a spark-generated cavitation bubble in stationary tap water is significantly increased by an applied magnetic field.

Perel'man and Govorskii ${ }^{8}$ showed, by using a vibratory apparatus in a liquid metal (probably sodium), that the weight loss of a metal specimen due to cavitation erosion can be significantly reduced by a magnetic field. A similar experiment was conducted by Kamiyama et al. ${ }^{9}$ in tap water. Although the result is contrary to that of Perel'man and Govorskii, it is clearly shown that the magnetic field can give visible changes to the dynamics of cavitation cloud and erosion area even in tap water. Kamiyama and Yamasaki ${ }^{10}$ also investigated the effect of a magnetic field on hydraulic cavitation of flowing mercury by using two types of venturi tubes which have a step expansion and a gradual expansion, respectively. They found that the inception of cavitation in the case of step expansion is significantly influenced by a magnetic field.

Hammitt et al. ${ }^{11}$ also investigated the effect of a magnetic field on cavitation inception by using a vibratory apparatus in tap water. However, they could not find any difference in threshold pressure-amplitude for cavitation inception. Another piece of evidence on the effect of a magnetic field on bubble dynamics was given by the experiment of Wong et al. ${ }^{12}$ in which the effect of a magnetic field on the growth of gas bubbles supplied through an aperture was investigated. They showed that if the magnetic field is applied perpendicular to the direction of gravity, the bubble departure frequency from a plate in a liquid metal can be significantly reduced.

There are other situations in which bubble dynamics is influenced by a magnetic field. An electromagnetic force is applied during the solidification of alloys for grain refinement and degassing. Vivès ${ }^{13}$ showed that, when an alloy is solidified in the presence of well-developed cavitation induced by a time-varying electromagnetic force, a very fine 


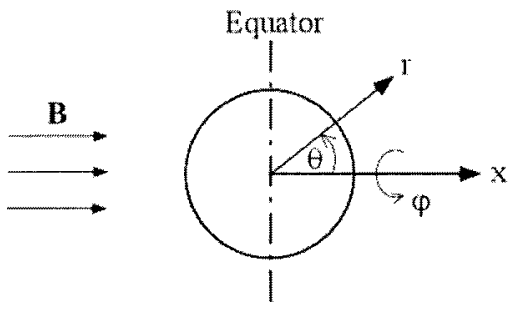

FIG. 1. The coordinate system adopted in this study.

and homogeneous microstructure can be obtained. Another example is found in the continuous casting process of steel. Inert argon gas is injected in the molten steel to capture impurities, while a magnetic field is applied to stabilize the flow of molten steel in the mold.

As described above, numerous problems are associated with the dynamics of bubbles in a magnetic field. However, very few theoretical results are known for the influence of the magnetic field on the dynamics of bubbles in conducting fluids, especially on a cavitation and boiling bubble. Wong et al. ${ }^{12}$ studied the effect of a magnetic field on the deformation of a bubble attached to a wall by using spheroidal approximation. However, authors did not provide a complete analysis of the magnetic-field effects on the bubble dynamics in conducting fluids. The purpose of the present work, therefore, is to investigate the effects of a uniform magnetic field on a growing or collapsing bubble in a weakly viscous conducting fluid.

\section{PROBLEM FORMULATION}

In the present work, we are concerned with the axisymmetric dynamics of a bubble in a weakly viscous, incompressible, and electrically conducting fluid subject to a uniform magnetic field, as shown in Fig. 1. The external fluid has the density $\rho$, the kinematic viscosity $\nu$, the conductivity $\sigma$, and the magnetic permeability $\mu$. All these material properties are assumed to be uniform in the external fluid phase. The interior of the bubble is assumed to be filled with a vapor and a permanent gas of low density so that the effect of the fluid motion inside the bubble on the external flow field can be neglected. The surface of the bubble is assumed to be characterized by a uniform surface tension $\gamma$.

The magnetic Reynolds number $R m=\sigma \mu l_{c} u_{c}$, where $l_{c}$ and $u_{c}$ are the characteristic length and the velocity scales, is assumed to be sufficiently small. If interior gas phase and exterior liquid phase are assumed to be nonmagnetizable, both phases have the same magnetic permeability. In this case, the magnetic field is not perturbed by the presence of a bubble, as long as the magnetic field associated with induced current due to bubble motion is neglected.

Assumption of the fluids being nonmagnetizable does not impose any severe limitation on the range of application of the present analysis. This is because many practically important fluids, such as water, air, sodium, and potassium, belong to this category. ${ }^{14}$

The main purpose of the present analysis is to firstly elucidate the effect of a magnetic field on the dynamics of a bubble. Thus, the effects of other parameters such as the compressibility of the surrounding fluid, condensation, evaporation, and heat transfer, which in some cases become important, are neglected. Furthermore, the effect of a solid wall is not considered. As is well known, the existence of a wall can significantly change the dynamics of a bubble, especially when the distance from the center of the bubble to the wall is in the order of the maximum bubble radius. ${ }^{15}$ However, the presence of the wall can only complicate the analysis of the problem without aiding to the main objective of the present investigation.

The spherical coordinate system $(r, \theta, \varphi)$ is adopted and the unit vectors in $r, \theta$, and $\varphi$ directions are denoted by $\mathbf{e}_{r}$, $\mathbf{e}_{\theta}$, and $\mathbf{e}_{\varphi}$, respectively. The uniform magnetic field is applied in the direction parallel to the positive $x$ axis as

$$
\mathbf{B}_{0}=B_{0} \mathbf{e}_{x}=B_{0}\left(\mathbf{e}_{r} \cos \theta-\mathbf{e}_{\theta} \sin \theta\right),
$$

where $\mathbf{e}_{x}$ denotes the unit vector parallel to the $x$ axis. The flow exterior to the bubble satisfies the following momentum equation: ${ }^{16}$

$$
\frac{\partial \mathbf{u}}{\partial t}+\Omega \times \mathbf{u}=-\nabla\left(\frac{p}{\rho}+\frac{1}{2} u^{2}\right)+\frac{\mathbf{F}_{L}}{\rho}-\nu \nabla \times \Omega,
$$

where $\mathbf{u}=\left(u_{r}, u_{\theta}, 0\right)$ denotes the fluid velocity, $p$ the dynamic pressure and $\Omega(=\nabla \times \mathbf{u})$ the vorticity, respectively. The Lorentz force $\mathbf{F}_{L}$ is given by

$$
\mathbf{F}_{L}=\mathbf{J} \times \mathbf{B},
$$

where $\mathbf{J}$ is the electric current density. From the Maxwell equations and Ohm's law, the current density becomes

$$
\mathbf{J}=\frac{1}{\mu} \nabla \times \mathbf{B}=\sigma(\mathbf{E}+\mathbf{u} \times \mathbf{B}),
$$

where $\mathbf{E}$ represents the electric field.

In the momentum equation, (2), the force due to the gravitational acceleration is neglected. It can be shown without difficulty that the ratio of the gravity force with respect to the electromagnetic force becomes $\rho g /\left(\sigma \dot{R} B_{0}^{2}\right)$ in which $g$ represents the gravitational acceleration, and $\dot{R}$ the radial velocity of the bubble wall. The ratio is much less than unity for typical liquid metals such as mercury, sodium, molten steel, and liquid aluminum, under a normal condition of, say, $B_{0}=1$ tesla and $\left|p_{\text {vap }}-p_{\infty}\right|=1$ bar. Here, $p_{\text {vap }}$ is the vapor pressure of the surrounding fluid, and $p_{\infty}$ the constant pressure at a distance large enough to be independent of bubble motion.

The shape of an axisymmetric bubble can be represented by the function $\Psi(r, \theta, t)$ defined as

$$
\Psi(r, \theta, t)=r-\zeta(\theta, t)=0 .
$$

At the bubble surface, the following kinematic condition and the normal stress conditions should be satisfied:

$$
\begin{aligned}
& u_{r}=\frac{\partial \zeta}{\partial t}+\frac{u_{\theta}}{r} \cdot \frac{\partial \zeta}{\partial \theta}, \\
& {\left[\left[\mathbf{n}\left(\mathbf{n} \cdot \mathbf{T}^{e m}\right)\right]\right]+\left[\left[\mathbf{n} \cdot\left(\mathbf{n} \cdot \mathbf{T}^{h}\right)\right]\right]=\gamma \nabla \cdot \mathbf{n},}
\end{aligned}
$$

where the symbol $[[(\cdot)]]$ denotes the external quantity minus the internal quantity, and $\mathbf{n}$ the outward unit normal vector 
on the bubble surface. In (7), $\mathbf{T}^{e m}$ and $\mathbf{T}^{h}$ represent the electromagnetic and the hydrodynamic stress tensors, and they are given by

$$
\begin{aligned}
& \mathbf{T}^{e m}=e\left[\mathbf{E E}-\frac{1}{2} E^{2} \mathbf{I}\right]+\mu\left[\mathbf{H H}-\frac{1}{2} H^{2} \mathbf{I}\right], \\
& \mathbf{T}^{h}=-p \mathbf{I}+\rho \nu\left[\nabla \mathbf{u}+\nabla \mathbf{u}^{T}\right],
\end{aligned}
$$

where $\mathbf{H}=\mathbf{B} / \mu$ and $e$ is the electric permittivity of the surrounding fluid.

As we can see in (7), the applied magnetic field can affect the bubble dynamics in two ways. One is the direct contribution denoted by $\mathbf{T}^{e m}$ and the other is the indirect contribution through the change in $\mathbf{T}^{h}$. It can be shown without difficulty that the electric field $\mathbf{E}$ in (4) vanishes to the first-order effect of the magnetic field, under the assumption of no free electric charge inside the conducting fluid medium (i.e., $\nabla \cdot \mathbf{J}=0$ ). Since $\mathbf{E}=\mathbf{0}$ and the magnetic permeabilities are the same both inside and outside the bubble, we can see from (8) that $\left[\left[\mathbf{T}^{e m}\right]\right]$ vanishes.

The normal stress condition (7) at the bubble surface is reduced to the following form:

$$
p_{\text {in }}-p_{\text {out }}+\left.\mathbf{n} \cdot \mathbf{n} \cdot \tau\right|_{\text {out }}=\gamma \nabla \cdot \mathbf{n} .
$$

Here $\tau$ denotes the viscous stress tensor. The pressure inside bubble $p_{\text {in }}$ can be represented as

$$
p_{\text {in }}=p_{\text {vap }}+p_{\text {go }}\left(\frac{R_{o}}{R}\right)^{3 \kappa},
$$

in which $p_{\text {go }}$ is the initial gas pressure, $R_{o}$ the initial radius of the bubble, $R$ the radius of the bubble, and $\kappa$ the specific heat ratio.

From the above argument, we have also seen that the Lorentz force is given approximately by $\mathbf{F}_{L}=\sigma\left(\mathbf{u} \times \mathbf{B}_{0}\right)$ $\times \mathbf{B}_{0}$. This Lorentz force modifies the flow field outside the bubble, and this flow-field change results in the change of hydrodynamic stress at the bubble surface. In the following section, the effect of the magnetic field on the hydrodynamic stress at the bubble surface will be discussed. Before proceeding further, we first want to derive an equation for modification of the fluid velocity due to the magnetic field.

For convenience, we nondimensionalize the equations by introducing the following nondimensional variables:

$$
\begin{gathered}
r^{*}=\frac{r}{R_{o}}, \quad t^{*}=\frac{\left|\left(p_{\mathrm{vap}}-p_{\infty}\right) / \rho\right|^{1 / 2} t}{R_{o}}, \\
\mathbf{u}^{*}=\frac{\mathbf{u}}{\left|\left(p_{\mathrm{vap}}-p_{\infty}\right) / \rho\right|^{1 / 2}},
\end{gathered}
$$

where the asterisk denotes the dimensionless quantities. Hereafter, the asterisk is dropped for convenience's sake. For later usage, the magnetic interaction parameter $N$, the Reynolds number $R e$, and the Weber number We are introduced and defined, respectively, by

$$
\begin{aligned}
& N=\frac{\sigma B_{o}^{2} R_{o}}{\left|\rho\left(p_{\mathrm{vap}}-p_{\infty}\right)\right|^{1 / 2}}, \\
& R e=\frac{R_{o}\left|\left(p_{\mathrm{vap}}-p_{\infty}\right) / \rho\right|^{1 / 2}}{\nu}, \quad W e=\frac{\left|p_{\mathrm{vap}}-p_{\infty}\right| R_{o}}{\gamma} .
\end{aligned}
$$

The momentum equation can be rewritten in the nondimensional form as

$$
\frac{\partial \mathbf{u}}{\partial t}+\Omega \times \mathbf{u}=-\nabla \Phi+N\left(\mathbf{u} \times \mathbf{e}_{x}\right) \times \mathbf{e}_{x}-\frac{1}{R e} \nabla \times \Omega,
$$

where $\Phi=p+\mathbf{u} \cdot \mathbf{u} / 2$. If the curl and divergence of (14) are taken, respectively, the following vorticity and pressure equations are obtained:

$\frac{\partial \Omega}{\partial t}+\nabla \times(\Omega \times \mathbf{u})$

$$
=N \nabla \times\left[\left(\mathbf{u} \times \mathbf{e}_{x}\right) \times \mathbf{e}_{x}\right]-\frac{1}{R e} \nabla \times \nabla \times \Omega,
$$

$\nabla^{2} \Phi=N \nabla \cdot\left[\left(\mathbf{u} \times \mathbf{e}_{x}\right) \times \mathbf{e}_{x}\right]-\nabla \cdot(\Omega \times \mathbf{u})$.

The velocity vector can be decomposed into two parts which describe the potential flow field and the rotational flow field, respectively as

$$
\mathbf{u}=\nabla \phi+\widetilde{\mathbf{u}}=\nabla \phi+\nabla \times \mathbf{A} .
$$

Here, $\phi$ is the velocity potential, and the vector potential $\mathbf{A}$ represents the velocity field due to the rotational component of the fluid motion. This vector potential has only $\varphi$-directional component, which is denoted by $A_{\varphi}$, owing to the axisymmetry of the flow assumed in this study. This function is related to the stream function $\psi$ and the $r, \theta$ components of the velocity vector as ${ }^{17}$

$$
\begin{aligned}
& A_{\varphi}=\frac{\psi}{r \sin \theta}, \\
& \tilde{u}_{r}=\frac{1}{r \sin \theta} \frac{\partial}{\partial \theta}\left(\sin \theta A_{\varphi}\right), \quad \tilde{u}_{\theta}=-\frac{1}{r} \frac{\partial\left(r A_{\varphi}\right)}{\partial r} .
\end{aligned}
$$

The vorticity also has only $\varphi$-directional component $\omega$ (i.e., $\Omega=(0,0, \omega))$ which is related to $A_{\varphi}$ as

$$
\omega=(\nabla \times \widetilde{\mathbf{u}})_{\varphi}=-\left(\nabla^{2} A_{\varphi}-\frac{A_{\varphi}}{r^{2} \sin ^{2} \theta}\right),
$$

where the subscript $\varphi$ denotes the $\varphi$-directional component.

\section{HYDRODYNAMIC STRESS CHANGE DUE TO A MAGNETIC FIELD}

It is assumed that the axisymmetric bubble dynamics can be described by the shape function

$$
\begin{aligned}
r=\zeta(\theta, t) & =R(t)+\varepsilon g(\theta, t) \\
& =R(t)+\varepsilon \sum_{n=2}^{\infty} a_{n}(t) P_{n}(\cos \theta),
\end{aligned}
$$

where $\varepsilon$ is a small perturbation parameter representing the distortion of the bubble from the spherical shape, and $P_{n}(\cos \theta)$ denote the Legendre polynomials. The case of $n$ $=1$ represents the translation of the bubble. Since the magnetic field has no influence on this mode of motion, as explained below (49), $n=1$ is excluded from the series given in (20).

If it is assumed that $R e \gg 1$, the effect of viscosity is confined to a thin boundary layer adjacent to the bubble sur- 
face. Thus, the flow domain can be divided into two regions. One is the inviscid region where the viscous effect can be neglected, and the other is the thin boundary layer near the bubble wall where the viscous effect should be considered. The effect of viscosity can be included by adding the viscous pressure correction and the viscous stress term in the normal stress balance, while the external flow field is computed using the inviscid approximation.

For the inviscid region, the governing equations for the flow field are given by

$$
\nabla \cdot \mathbf{u}=0, \quad \frac{\partial \mathbf{u}}{\partial t}+\Omega \times \mathbf{u}=-\nabla \Phi+N\left(\mathbf{u} \times \mathbf{e}_{x}\right) \times \mathbf{e}_{x} .
$$

Here we perform a perturbation analysis based on the assumptions of $0<\varepsilon \ll 1$ and $0<N \ll 1$ implying small disturbances by the fluid motion and electromagnetic field, respectively. The perturbation expansion of $\mathbf{u}$ and $p$ are in the form of

$$
\mathbf{u}=\mathbf{u}_{0}+\varepsilon \mathbf{u}_{1}+N \mathbf{u}_{m}+\cdots, \quad p=p_{0}+\varepsilon p_{1}+N p_{m}+\cdots .
$$

The first terms in the expressions $\left(\mathbf{u}_{0}, p_{0}\right)$, describe the potential flow field due to the radial motion of the bubble wall. The second terms $\left(\varepsilon \mathbf{u}_{1}, \varepsilon p_{1}\right)$ represent the potential flow field due to the distortion of the bubble from the spherical shape. The third terms, $\left(N \mathbf{u}_{m}, N p_{m}\right)$ represent the rotational flow due to the electromagnetic force. Thus the vorticity in (21) can be represented as

$$
\Omega=\nabla \times \mathbf{u}=N \nabla \times \mathbf{u}_{m} \equiv N \Omega_{m},
$$

and $\Phi$ can be expanded as

$$
\begin{aligned}
\Phi= & \left(p_{0}+\frac{1}{2} \mathbf{u}_{0} \cdot \mathbf{u}_{0}\right)+\varepsilon\left(p_{1}+\mathbf{u}_{0} \cdot \mathbf{u}_{1}\right)+N\left(p_{m}+\mathbf{u}_{0} \cdot \mathbf{u}_{m}\right) \\
& +\cdots
\end{aligned}
$$

On the other hand, the kinematic condition (6), which should be satisfied at $r=\zeta=R+\varepsilon g$, can be transformed to an equivalent condition at $r=R$ as

$$
\begin{aligned}
u_{0 r}+\varepsilon u_{1 r}+N u_{m r}+\varepsilon g \frac{\partial u_{0 r}}{\partial r}+\cdots= & \dot{R}+\varepsilon \frac{\partial g}{\partial t} \\
& +\varepsilon \frac{u_{0 \theta}}{R} \frac{\partial g}{\partial \theta}+\cdots .
\end{aligned}
$$

Now, we have the following continuity equation, momentum equation, and kinematic boundary condition, for the problems of $O(1), O(\varepsilon)$, and, $O(N)$, respectively, for the region $r \geqslant R$ :

$$
\begin{array}{ll}
O(1): & \nabla \cdot \mathbf{u}_{0}=0, \quad \frac{\partial \mathbf{u}_{0}}{\partial t}+\frac{1}{2} \nabla\left(\mathbf{u}_{0} \cdot \mathbf{u}_{0}\right)=-\nabla p_{0}, \\
u_{0 r}=\dot{R} \text { at } r=R, \\
O(\varepsilon): \quad \nabla \cdot \mathbf{u}_{1}=0, \quad \frac{\partial \mathbf{u}_{1}}{\partial t}+\nabla\left(\mathbf{u}_{1} \cdot \mathbf{u}_{0}\right)=-\nabla p_{1}, \\
u_{1 r}=\frac{\partial g}{\partial t}-g \frac{\partial u_{0 r}}{\partial r}+\frac{u_{0 \theta}}{R} \frac{\partial g}{\partial \theta} \text { at } r=R,
\end{array}
$$

$$
\begin{array}{ll}
O(N): \quad & \nabla \cdot \mathbf{u}_{m}=0, \\
& \frac{\partial \mathbf{u}_{m}}{\partial t}+\Omega_{m} \times \mathbf{u}_{0}=-\nabla \Phi_{m}+\left(\mathbf{u}_{0} \times \mathbf{e}_{x}\right) \times \mathbf{e}_{x}, \\
& u_{m r}=0 \text { at } r=R,
\end{array}
$$

where $\Phi_{m}=p_{m}+\mathbf{u}_{0} \cdot \mathbf{u}_{m}$. The $O(1)$ and $O(\varepsilon)$ problems yield the solutions ${ }^{18}$

$$
\begin{aligned}
& \mathbf{u}_{0}=\nabla \phi_{0}, \quad \phi_{0}=-\frac{R^{2} \dot{R}}{r}, \\
& \mathbf{u}_{1}=\nabla \phi_{1}, \\
& \phi_{1}=-\sum_{n=1}^{\infty} \frac{r}{n+1}\left(\frac{R}{r}\right)^{n+2}\left(\dot{a}_{n}+\frac{2 a_{n} \dot{R}}{R}\right) P_{n}(\cos \theta) .
\end{aligned}
$$

The $O(N)$ problem can be solved as follows. The vorticity and the pressure equations for this order are

$$
\begin{aligned}
& \frac{\partial \Omega_{m}}{\partial t}+\nabla \times\left(\Omega_{m} \times \mathbf{u}_{0}\right)=\nabla \times\left[\left(\mathbf{u}_{0} \times \mathbf{e}_{x}\right) \times \mathbf{e}_{x}\right], \\
& \nabla^{2} \Phi_{m}=\nabla \cdot\left[\left(\mathbf{u}_{0} \times \mathbf{e}_{x}\right) \times \mathbf{e}_{x}\right]-\nabla \cdot\left(\Omega_{m} \times \mathbf{u}_{0}\right) .
\end{aligned}
$$

When the vorticity vector is given as $\Omega_{m}=\left(0,0, \omega_{m}\right)$, the vorticity equation for the perturbed flow due to the electromagnetic force is obtained as

$$
\frac{\partial \omega_{m}}{\partial t}+r u_{0 r} \frac{\partial}{\partial r}\left(\frac{\omega_{m}}{r}\right)=\frac{u_{0 r}}{r} P_{2}^{1}(\cos \theta),
$$

where $u_{0 r}=R^{2} \dot{R} / r^{2}$ and $P_{2}^{1}(\cos \theta)=3 \cos \theta \sin \theta$. Under the assumption that the vorticity is initially zero everywhere, i.e., $\omega(r, \theta, 0)=0$, the solution of (33) can be obtained as

$$
\omega_{m}(r, \theta, t)=\left[\frac{r}{\left(r^{3}-R^{3}+1\right)^{1 / 3}}-1\right] P_{2}^{1}(\cos \theta) .
$$

It is noteworthy that the vorticity distribution is dependent only on the bubble radius $R$ and independent of the rate of radius change $\dot{R}$. This result may seem to be a little strange at first glance. However, it is possible, because the viscous effect is neglected. Furthermore, we can see from (33) that

$$
\frac{D}{D t}\left(\frac{\omega_{m}}{r}\right)=\frac{\partial}{\partial t}\left(\frac{\omega_{m}}{r}\right)+\mathbf{u}_{0} \cdot \nabla\left(\frac{\omega_{m}}{r}\right)=\frac{R^{2} \dot{R}}{r^{4}} P_{2}^{1}(\cos \theta) .
$$

The above equation indicates that $D\left(\omega_{m} / r\right) / D t$ changes its sign when $\dot{R}$ changes sign.

Now the perturbed velocity field due to the magnetic field, $\mathbf{u}_{m}$, can be obtained by computing $A_{\varphi}$ in (19) as (note $\widetilde{\mathbf{u}}=N \mathbf{u}_{m}$ and $\omega=N \omega_{m}$ to the first order of $N$ )

$$
\nabla^{2} A_{\varphi m}-\frac{A_{\varphi m}}{r^{2} \sin ^{2} \theta}=\left[1-\frac{r}{Z(r, R)^{1 / 3}}\right] P_{2}^{1}(\cos \theta),
$$

where $A_{\varphi}=N A_{\varphi m}$ and $Z(r, R)=r^{3}-R^{3}+1$. From the kinematic boundary condition that $\tilde{u}_{r}=N u_{m r}=0$ at $r=R$, and the condition at infinity, the function $A_{\varphi m}$ should satisfy the following boundary conditions: 


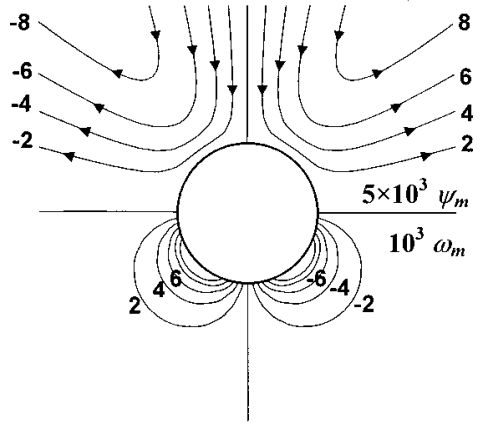

(a)

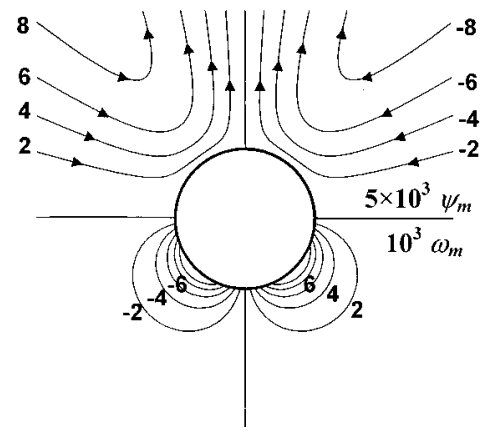

(b)

FIG. 2. The streamlines and vorticity contours of the perturbed flow due to a magnetic field: (a) growing bubble $(R=1.01)$; (b) collapsing bubble $(R$ $=0.99$ ).

$$
\begin{aligned}
& A_{\varphi m}=0 \text { at } r=R, \\
& A_{\varphi m} \rightarrow 0 \text { as } r \rightarrow \infty .
\end{aligned}
$$

The solution of Eq. (35) has the following form:

$$
A_{\varphi m}(r, \theta, t)=f(r, t) P_{2}^{1}(\cos \theta)+\sum_{n=1}^{\infty} \frac{b_{n}}{r^{n+1}} P_{n}^{1}(\cos \theta) \text {. }
$$

The binomial expansion of $1-r / Z(r, R)^{1 / 3}$ is introduced to find $f(r, t)$, and then the boundary conditions of (36) are used to determine the unknown coefficients $b_{n}$, so that

$$
A_{\varphi m}(r, \theta, t)=\left[f(r, t)-f(R)\left(\frac{R}{r}\right)^{3}\right] P_{2}^{1}(\cos \theta),
$$

in which, for $R<1$

$$
\begin{aligned}
f(r, t)= & \frac{r^{2}}{5} \sum_{n=1}^{\infty}\left(\begin{array}{c}
\frac{1}{3} \\
n
\end{array}\right)(-1)^{n}\left\{\frac{1}{5}\left(\frac{S}{Z}\right)^{5 / 3}{ }_{2} F_{1}\left(\frac{5}{3}, \frac{8}{3}-n ; \frac{8}{3} ; \frac{r^{3}}{Z}\right)\right. \\
& \left.+\frac{1}{3 n}\left(\frac{S}{Z}\right)^{n}{ }_{2} F_{1}\left(n, 1 ; n+1 ; \frac{S}{Z}\right)\right\},
\end{aligned}
$$

and for $R>1$

$$
\begin{aligned}
f(r, t)= & \frac{-2 r^{5}+\left(2 r^{3}-3 S\right) Z^{2 / 3}}{50 r^{3}} \\
& -\frac{1}{45} \frac{S}{r}{ }_{3} F_{2}\left(1,1, \frac{4}{3} ; 2,2 ;-\frac{S}{r^{3}}\right),
\end{aligned}
$$

where ${ }_{2} F_{1}$ and ${ }_{3} F_{2}$ represent hypergeometric functions, ${ }^{19}$ and $S \equiv 1-R^{3}$. When $R=1, f(r, t)$ becomes null.
The stream function for the magnetically induced flow can be obtained from (18a) and (37). In Fig. 2, the streamlines $\left(\psi_{m}\right)$ and vorticity contours $\left(\omega_{m}\right)$ are shown for $R$ $=1.01$ and $R=0.99$ (note $\psi=N \psi_{m}$ ). It is found that the flow direction changes as the value of $R$ decreases from greater than unity to less than unity. For a growing bubble $(R>1)$, the perturbed flow by the magnetic field is incoming near the equator $\left(\theta=\frac{1}{2} \pi\right)$ and outgoing near the poles $(\theta=0, \pi)$. On the other hand, in the case of a collapsing bubble $(R<1)$, the flow direction is reversed. Therefore, the magnitude of the overall velocity in the inviscid region to the first order of $\varepsilon$ and $N$, i.e., $\left(\mathbf{u} \cong \mathbf{u}_{0}+\varepsilon \mathbf{u}_{1}+N \mathbf{u}_{m}\right)$, becomes smaller near the equator than near the poles, regardless of the direction of bubble wall motion.

Next, the pressure correction due to the magnetic field is obtained to determine the bubble shape by using the normal stress balance. Since $\Phi_{m}=p_{m}+\mathbf{u}_{0} \cdot \mathbf{u}_{m}$ for which we know $\mathbf{u}_{0}$ and $\mathbf{u}_{m}, p_{m}$ can be obtained if $\Phi_{m}$ is known. From (32), we have

$$
\nabla^{2} \Phi_{m}=\nabla \cdot\left[u_{0 r}\left(\mathbf{e}_{r} \times \mathbf{e}_{x}\right) \times \mathbf{e}_{x}\right]-\nabla \cdot\left(\omega_{m} u_{0 r} \mathbf{e}_{\theta}\right) .
$$

We substitute $\omega_{m}$ of (34) into the above equation to have the reduced pressure equation

$$
\nabla^{2} \Phi_{m}=\frac{4 u_{0 r}}{r}\left[1-\frac{3}{2} \frac{r}{Z(r, t)^{1 / 3}}\right] P_{2}(\cos \theta) .
$$

The solution of the above equation can be expressed as follows:

$$
\Phi_{m}(r, \theta, t)=h(r, t) P_{2}(\cos \theta)+\sum_{n=0}^{\infty} \frac{d_{n}}{r^{n+1}} P_{n}(\cos \theta)
$$

The power-series expansion of $1-\frac{3}{2} \cdot r / Z(r, t)^{1 / 3}$ is introduced to find $h(r, t)$. The unknown coefficients $d_{n}$ can be determined by using the $r$-directional momentum equation of (14) to the $O(N)$. Then $\Phi_{m}$ becomes

$$
\begin{aligned}
\Phi_{m}(r, \theta, t)= & \frac{2}{3} \frac{R^{2} \dot{R}}{r}+R \dot{R}\left\{\frac{h(r, t)}{R \dot{R}}-\frac{2}{r^{3} R} \frac{\partial}{\partial R}\left[R^{3} f(R)\right]\right\} \\
& \times P_{2}(\cos \theta),
\end{aligned}
$$

in which, for $R<1$

$$
\begin{aligned}
h(r, t)= & \frac{R^{2} \dot{R}}{3 r}+\frac{R^{2} \dot{R}}{r} \sum_{n=1}^{\infty}\left(\begin{array}{c}
\frac{1}{3} \\
n
\end{array}\right) \\
& \times(-1)^{n}\left\{\frac{3}{5}\left(\frac{S}{Z}\right)^{2 / 3}{ }_{2} F_{1}\left(\frac{2}{3}, \frac{5}{3}-n ; \frac{5}{3} ; \frac{r^{3}}{Z}\right)\right. \\
& \left.+\frac{2}{5(n+1)}\left(\frac{S}{Z}\right)^{n}{ }_{2} F_{1}\left(n, 1 ; n+2 ; \frac{S}{Z}\right)\right\},
\end{aligned}
$$

and for $R>1$

$$
h(r, t)=-\frac{2}{3} \frac{R^{2} \dot{R}}{r}+\frac{3}{5} \frac{Z^{5 / 3}-r^{5}}{r^{3} S} .
$$


Since $p_{m}=\Phi_{m}-u_{0 r} u_{m r}$, the electromagnetic correction of the pressure, to the first order of $N$, becomes

$$
\begin{aligned}
N p_{m}(r, \theta, t)= & N \frac{2}{3} \frac{R^{2} \dot{R}}{r}+N R \dot{R}\left\{\frac{h(r, t)}{R \dot{R}}\right. \\
& \left.-\frac{2}{r^{3} R} \frac{\partial}{\partial R}\left[R^{3} f(R)\right]\right\} P_{2}(\cos \theta) \\
& -\frac{6 N}{r}\left\{f(r, t)-f(R)\left(\frac{R}{r}\right)^{3}\right\} P_{2}(\cos \theta) .
\end{aligned}
$$

Thus, the electromagnetic correction of pressure at the bubble wall is

$$
N p_{m}(R, \theta)=\frac{2}{3} N R \dot{R}+N R \dot{R} Q(R) P_{2}(\cos \theta),
$$

in which, for $R<1$

$$
\begin{aligned}
Q(R)= & \frac{1}{3}+\sum_{n=1}^{\infty}\left(\begin{array}{c}
\frac{1}{3} \\
n
\end{array}\right)(-1)^{n}\left\{\frac{3 S^{2 / 3}}{5}{ }_{2} F_{1}\left(\frac{2}{3}, \frac{5}{3}-n ; \frac{5}{3} ; R^{3}\right)\right. \\
& +\frac{2 S^{n}}{5(n+1)}{ }_{2} F_{1}(n, 1 ; n+2 ; S) \\
& +\frac{2 R^{3} S^{2 / 3}}{5}{ }_{2} F_{1}\left(\frac{5}{3}, \frac{8}{3}-n ; \frac{8}{3} ; R^{3}\right) \\
& \left.-\frac{2 S^{n}}{3 n}{ }_{2} F_{1}(n, 1 ; n+1 ; S)\right\},
\end{aligned}
$$

and for $R>1$

$$
Q(R)=\frac{R^{2}+R-2}{3\left(R^{2}+R+1\right)}+\frac{2}{9} \frac{S}{R^{3}}{ }_{3} F_{2}\left(1,1, \frac{4}{3} ; 2,2 ;-\frac{S}{R^{3}}\right) .
$$

The function $Q(R)$ is shown in Fig. 3 for the cases of a growing bubble [Fig. 3(a)] and a collapsing bubble [Fig. 3(b)]. The function $Q$ is negative for all $R$ in the case of a growing bubble $(\dot{R}>0, R>1)$. On the contrary, $Q$ is positive in the case of a collapsing bubble $(\dot{R}<0, R<1)$. Consequently, the coefficient of $P_{2}(\cos \theta)$ in (44) is always negative for both cases. This means that the magnetically induced pressure has greater value near the equator of the bubble than near the poles, since $P_{2}=-1 / 2$ at $\theta=\pi / 2$ and $P_{2}=1$ at $\theta$ $=0, \pi$.

This result can be interpreted as follows. In the momentum equation, (14), the Lorentz force acting on fluid elements around a bubble can be expressed to the first order of $N$ as

$$
\mathbf{F}_{L}=-N u_{0 r}\left(\sin ^{2} \theta \mathbf{e}_{r}+\sin \theta \cos \theta \mathbf{e}_{\theta}\right) .
$$

It is evident that the magnitude of the $r$-directional force is greatest near the equator, and the radial direction of the force is always opposite to the direction of bubble wall motion. So, without considering the effect of magnetically induced flow, the pressure near the equator is increased for a growing bubble, and decreased for a collapsing bubble. However, the deceleration of the flow velocity, due to the magnetically

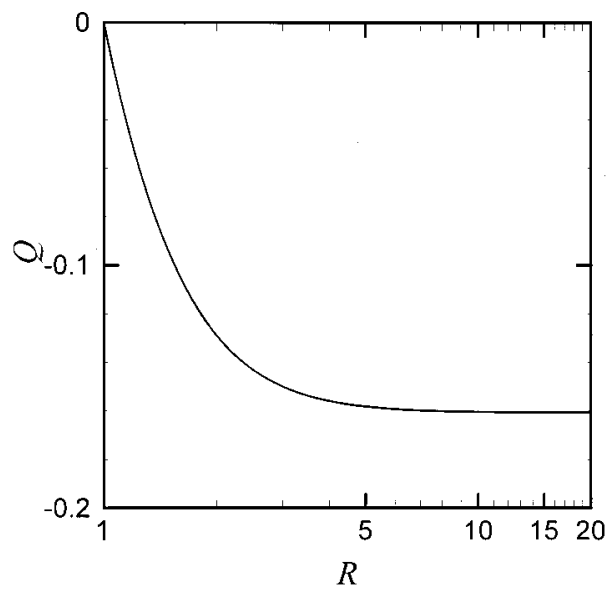

(a)

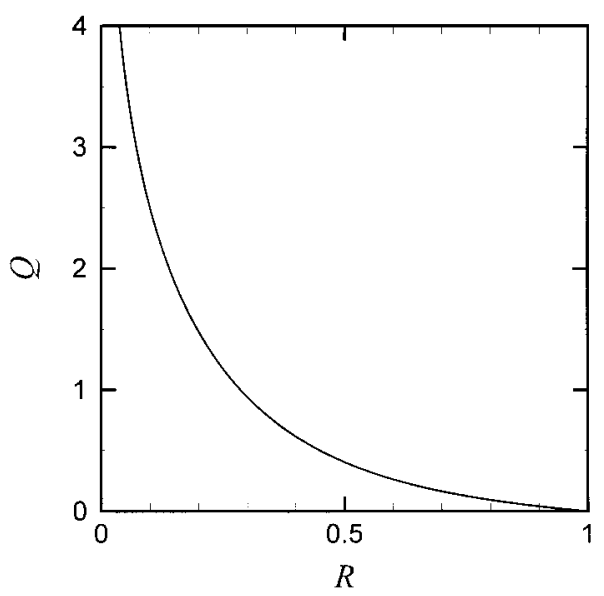

(b)

FIG. 3. The function $Q(R)$ defined in (45): (a) growing bubble $(\dot{R}>0)$; (b) collapsing bubble $(\dot{R}<0)$.

induced flow, is most noticeable near the equator (see Fig. 2). This will result in the increase of the pressure near the equator, regardless of the bubble wall motion. Thus, even in the case of a collapsing bubble, the pressure near the equator ( $\left.\theta=\frac{1}{2} \pi\right)$ has larger values than near the poles $(\theta=0, \pi)$.

\section{BUBBLE SHAPE}

The normal stress condition given by (10) at the bubble surface $\left[r=R(t)+\varepsilon \sum_{n=2}^{\infty} a_{n}(t) P_{n}(\cos \theta)\right]$ is given in dimensionless form as

$$
\begin{aligned}
p_{\text {in }} & \left(p_{\infty}+\frac{1}{R e} p_{\nu}+N p_{m}\right)+\left(\frac{\partial \phi}{\partial t}+\frac{1}{2} \nabla \phi \cdot \nabla \phi\right) \\
& +\frac{2}{R e} \frac{\partial^{2} \phi}{\partial r^{2}}=\frac{1}{W e} \nabla \cdot \mathbf{n},
\end{aligned}
$$

where $\phi=\phi_{0}+\varepsilon \phi_{1},(1 / R e) p_{\nu}$ the viscous pressure correction, and $N p_{m}$ the pressure correction due to the magnetically induced flow. The viscous pressure correction can be obtained, by analyzing the flow under the boundary layer assumption, as follows: ${ }^{20}$ 


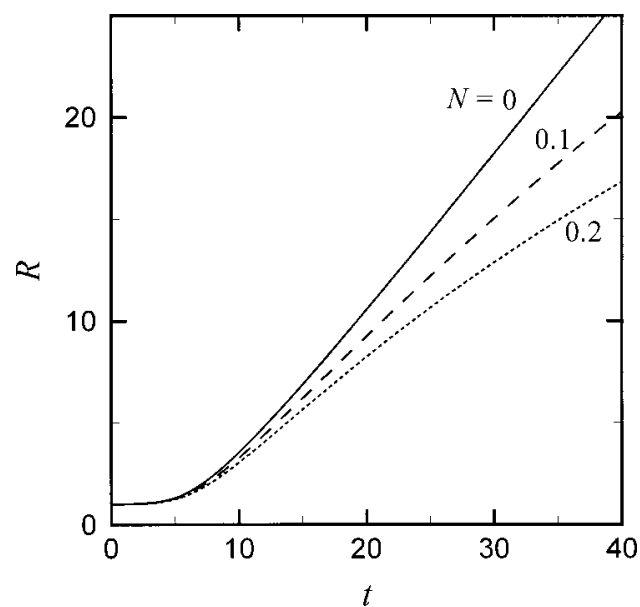

FIG. 4. The effect of a magnetic field on the volume mode of a growing bubble in the case of $R e=20, W e=2$.

$$
\begin{aligned}
\frac{1}{R e} p_{\nu}= & \frac{2 \varepsilon}{\operatorname{Re}} \sum_{n=2}^{\infty} \frac{n}{n+1} \\
& \times\left[\frac{(n+2) \dot{a}_{n}}{R}-\frac{(n-1) \dot{R} a_{n}}{R^{2}}\right] P_{n}(\cos \theta) .
\end{aligned}
$$

Substituting (29), (30), (44), and (47) into (46), we can obtain the equations of motion of the bubble wall. The procedure is straightforward and here we present only the results. For the spherically symmetric motion, the following equation is obtained:

$$
\begin{aligned}
R \ddot{R} & +\frac{3}{2} \dot{R}^{2}+\frac{4}{R e} \frac{\dot{R}}{R}+\frac{2}{3} N R \dot{R}+\frac{2}{W e} \frac{1}{R}-p_{\text {go }}\left(\frac{1}{R}\right)^{3 \kappa} \\
& =\frac{p_{\text {vap }}-P_{\infty}}{\left|p_{\text {vap }}-p_{\infty}\right|} .
\end{aligned}
$$

The fourth term in the left-hand side of the above equation represents the contribution from the magnetic field. When $N$ is null, the above equation becomes identical to the Rayleigh-Plesset equation. From (48), we can also see that the magnetic field exerts a damping force to the bubble. This result is consistent with the well-known fact that the static magnetic field retards the fluid motion when the magneticfield direction is not parallel to the flow direction.

For $n \geqslant 2$ (shape modes), the following equation is obtained:

$$
\begin{aligned}
\ddot{a}_{n}+ & {\left[\frac{3 \dot{R}}{R}+\frac{1}{R e} \frac{2(2 n+1)(n+2)}{R^{2}}\right] \dot{a}_{n}+(n-1) } \\
& \times\left[\frac{1}{W e} \frac{(n+1)(n+2)}{R^{3}}-\frac{\ddot{R}}{R}+\frac{2(n+2)}{R e} \frac{R}{R^{3}}\right] a_{n} \\
& =-\frac{N}{\varepsilon}(n+1) Q(R) \dot{R} \delta_{n 2} .
\end{aligned}
$$

The symbol $\delta_{n 2}$ is the Kronecker delta. To obtain a meaningful result, the small parameter for shape deformation $\varepsilon$ should be $O(N)$. Thus in this study, we set $\varepsilon=N$. The term in the right-hand side represents the contribution from the

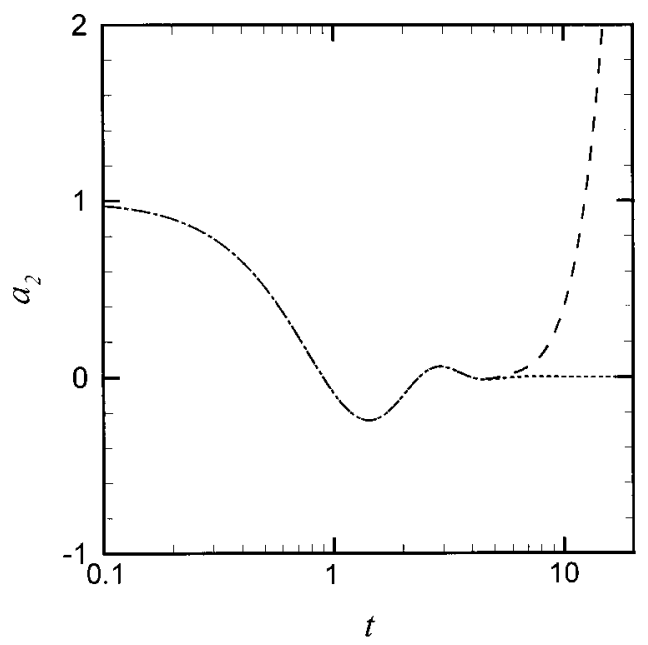

(a)

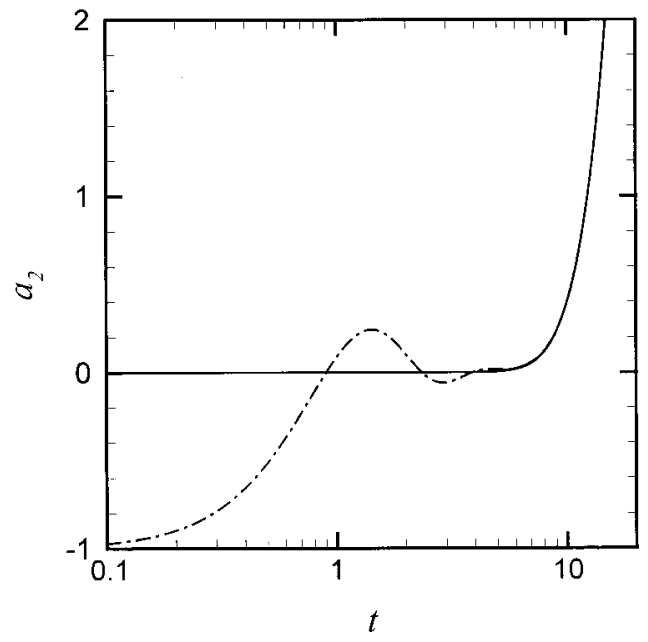

(b)

FIG. 5. The effect of a magnetic field on the $P_{2}$-mode shape change of a growing bubble in the case of $R e=20$ and $W e=2$ : (a) $\cdots \cdots N=0, a_{20}$ $=1 ;----N=0.1, a_{20}=1 ;$ (b) $-N=0.1, a_{20}=0 ;-\cdot-\cdot-\cdot-\cdot N$ $=0.1, a_{20}=-1$.

magnetic field. It should be noted that the term is always positive as explained below (45b). This means that the magnetic field results in a positive forcing term in the $P_{2}$-mode of bubble dynamics. The magnetic field has a destabilizing effect on bubble shape for both growing and collapsing bubbles, irrespective of the initial condition of bubble shape, which will be demonstrated in detail in this section. As explained below (48), however, the magnetic field has a damping effect on the volume mode of motion. A distinction should be made for the influence of the magnetic field on the two modes of bubble motion.

In Figs. 4 and 5, the solutions of $R$ and $a_{2}$ for a growing bubble are shown for the case of $R e=20, W e=2, \dot{R}(0)$ $=0.01$, and $\dot{a}_{2}(0)=0$. In the case of $N=0$ in Fig. 5(a), an initial disturbance of $a_{2}(0) \equiv a_{20}=1$ is given. For comparison with the results of Prosperetti and Seminara, ${ }^{21}$ the parameters are intentionally chosen to be identical to those of 


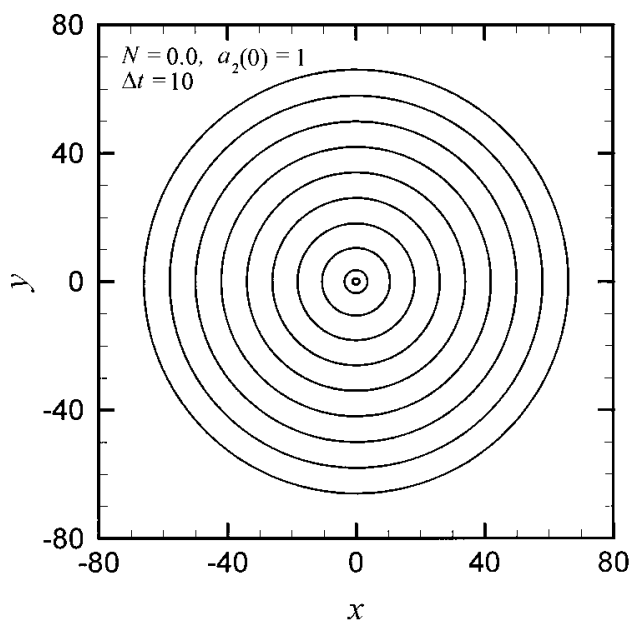

(a)

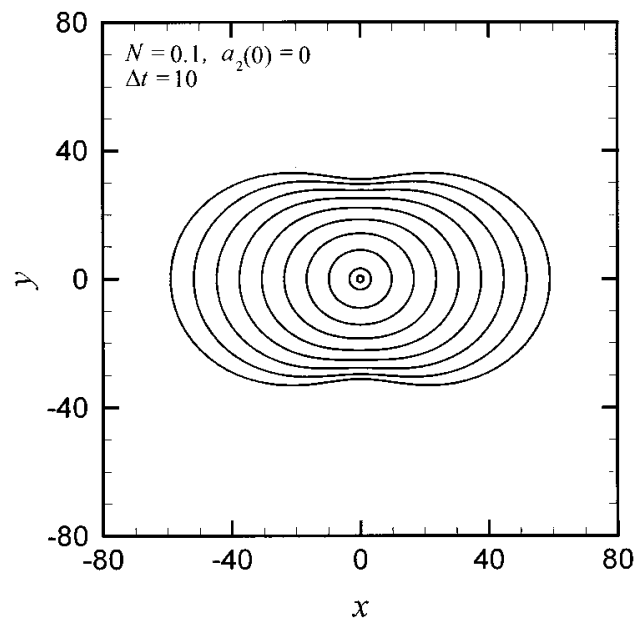

(b)

FIG. 6. The temporal evolution of the surface of a growing bubble in the case of $R e=20$ and $W e=2$.

Fig. 5 of Prosperetti and Seminara. ${ }^{21}$ It is confirmed that identical results to those of Prosperetti and Seminara are obtained in the case of no magnetic field.

The growth rate of the bubble for volume mode is reduced due to the damping effect of the magnetic field, as shown in Fig. 4. The shape mode is in general stable for a growing bubble in the absence of external disturbances, and therefore the initial disturbance soon diminishes, as shown in Fig. 5(a). That is, the shape of the bubble becomes spherical as shown in Fig. 6(a), in which $\Delta t$ denotes the time interval between each solid line.

To see the magnetic-field effect on the shape mode, the solution of $a_{2}(t)$ is obtained and shown in Fig. 5 for $N$ $=0.1$ with three different initial conditions of $a_{20}=-1,0$, and 1 . Before discussing the behavior of the $P_{2}$-shape mode in the magnetic field, the asymptotic form of (49) is discussed for better understanding of the results. If the initial growing velocity of the bubble is neglected, the growing speed of the bubble wall can be approximated as

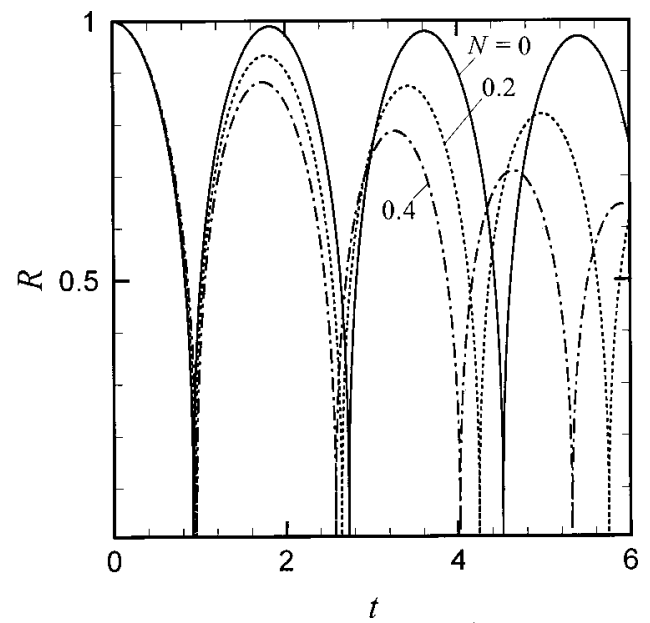

FIG. 7. The effect of a magnetic field on a collapsing bubble for the volume mode in the case of $R e=1000$ and $W e=2000$.

$$
\begin{aligned}
(\dot{R})^{2}= & \lambda^{2}\left(1-\frac{1}{R^{3}}\right)-\frac{1}{W e} \frac{1}{R}\left(1-\frac{1}{R^{2}}\right) \\
& +\lambda^{2} \frac{p_{\text {go }}}{(1-\kappa)} \frac{1}{R^{3}}\left[\left(\frac{1}{R}\right)^{3(\kappa-1)}-1\right], \quad \lambda^{2}=\frac{2}{3} .
\end{aligned}
$$

For a growing bubble, the velocity of the bubble wall asymptotically approaches the constant $\lambda$ as $R$ becomes larger. Then, for a large value of $R,(49)$ can be approximated to $\ddot{a}_{2}=-3 \lambda Q(R)$. For a large value of $R$, therefore, $a_{2}$ increases monotonically even without the initial disturbance [note $Q(R)<0$ for a growing bubble and see Fig. 5(b)].

Thus, the shape of the bubble is significantly deformed from the spherical shape as shown in Fig. 6(b) in which $a_{20}=0$. It is evident that the $P_{2}$-mode of a growing bubble becomes unstable, due to the magnetic field, regardless of initial conditions. As mentioned earlier, the magnetically induced pressure near the equator $\left(\theta=\frac{1}{2} \pi\right)$ has greater value than near the poles $(\theta=0, \pi)$ of the bubble. The uniform magnetic field, therefore, has a tendency to change the growing bubble into a prolate shape in the direction of the magnetic field and eventually make the bubble unstable, as illustrated in Fig. 6(b). If the volumes of two bubbles in Figs. 6(a) and 6(b) are compared, the bubble which is under the influence of a uniform magnetic field has smaller volume due to the damping effect of the magnetic field for volume mode of motion.

For illustration purposes, the time history of bubble shape in Fig. 6(b) is intentionally extended to the final stage of large distortion. The present linear analysis for a large distortion may not be valid. However, based on the previous study of collapsing bubbles obtained from the linear analysis, $^{22}$ it can be assumed that the linear theory may be extended even for large deformation. The accurate limit of the linear analysis can only be confirmed by properly conducted experiment.

In Fig. 7, the temporal evolution of radius of a collapsing and bouncing bubble is shown for three different interaction parameters. The other parameters are chosen to be identical to those of Fig. 2 of Prosperetti and Seminara, ${ }^{21}$ in which 


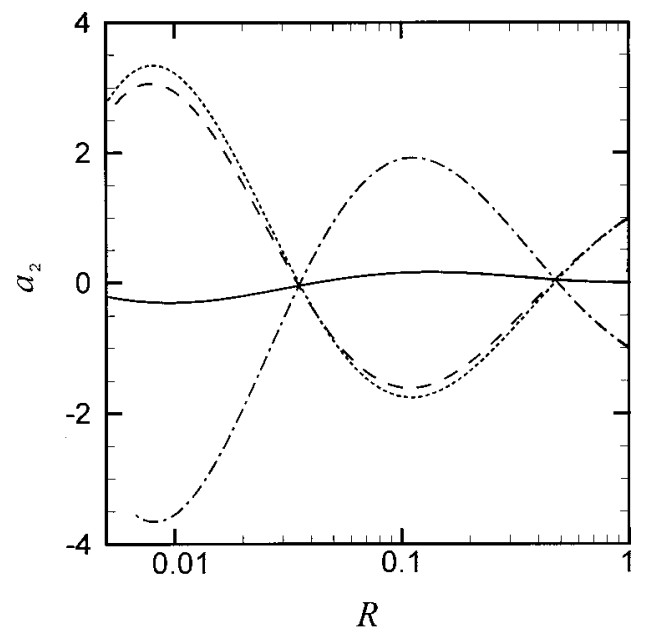

FIG. 8. The effect of a magnetic field on the $P_{2}$-mode shape change of a collapsing bubble in the case of $R e=1000$ and $W e=2000: \cdots \cdots N=0$, $a_{20}=1 ;-----N=0.1, a_{20}=1 ;-N=0.1, a_{20}=0 ;-\cdot-\cdot-\cdot-\cdot N$ $=0.1, a_{20}=-1$.

$R e=1000, W e=2000$, and $\dot{R}(0)=0$. As shown in Fig. 7, the magnetic force damps the volume mode of bubble motion.

The shape mode of a collapsing bubble exhibits more complicated behaviors than that of a growing bubble. To enhance understanding, an asymptotic analysis for a collapsing bubble is carried out. If the initial collapsing velocity of the bubble is neglected, the collapsing speed of the bubble wall can be approximated from (48) as

$$
(\dot{R})^{2}=\lambda^{2}\left(\frac{1}{R^{3}}-1\right)+\frac{1}{W e} \frac{1}{R}\left(\frac{1}{R^{2}}-1\right), \quad \lambda^{2}=\frac{2}{3} .
$$

Applying this to (49), the asymptotic form for sufficiently small $R$ obtained as

$$
\ddot{a}_{2}-\frac{3 \lambda}{R^{5 / 2}} \dot{a}_{2}+\frac{1}{R^{5}} a_{2}=-3 \dot{R} Q(R) .
$$

The above equation represents an equation of negatively damped forced oscillator. From (50), we can see also that the term $a_{2} / R^{5}$ becomes important when $R$ is very small.

In Fig. $8, a_{2}(t)$ obtained from (49) is plotted against $R(t)$ for a collapsing bubble $(R=1$ corresponds to $t=0$ and $R$ decreases as $t$ increases). The Reynolds and Weber numbers are identical to those in Fig. 7. As the case of Prosperetti and Seminara, it is assumed that $\dot{a}_{2}(0)=0$. In the case of $N=0$, an initial disturbance of $a_{20}=1$ is given [note that there is no forcing term in (50) in the case of no magnetic field]. As predicted by the asymptotic form, (50), a collapsing bubble is inherently unstable to external disturbances in the shape mode. The corresponding shape evolution is shown in Fig. 9(a).

To see the effect of a magnetic field on the shape mode of a collapsing bubble, we considered the case of $N=0.1$ with three different initial shapes of $a_{20}=-1,0$, and 1 (see Fig. 8). When an initial disturbance is given, the shape of the bubble becomes unstable regardless of the sign of the disturbance. When $a_{20}=0$ and $N=0.1, a_{2}$ has initially positive value $\left(0<a_{2}<1.16\right.$ for $\left.0.041<R \leqslant 1\right)$ due to the positive

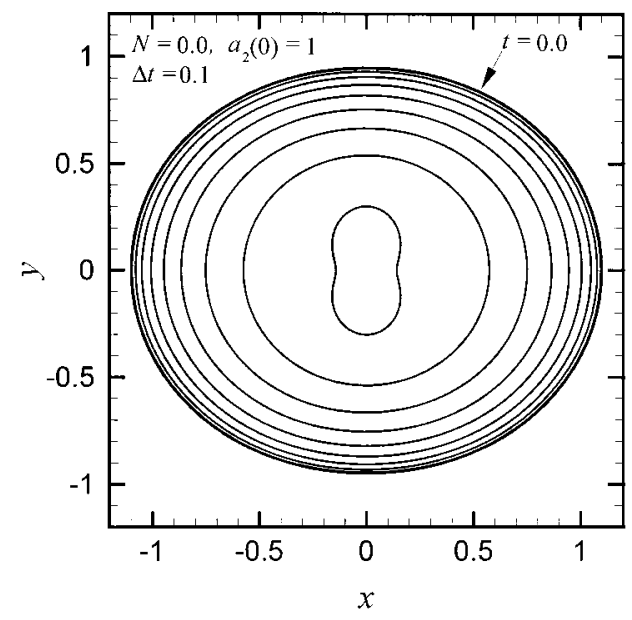

(a)

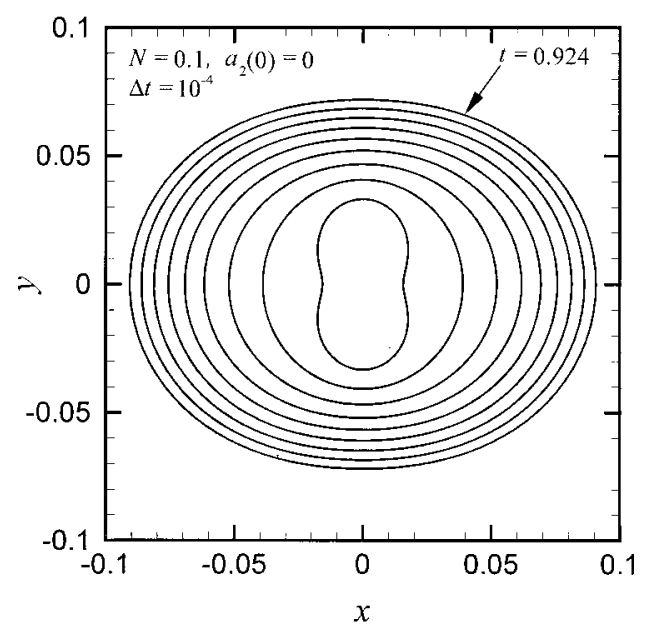

(b)

FIG. 9. The temporal evolution of the surface of a collapsing bubble in the case of $R e=1000$ and $W e=2000$.

forcing of the magnetic field [note $\dot{R}<0$ and $Q(R)>0$ ]. However, as the bubble size decreases further, it changes sign as shown in Fig. 8. As mentioned below (50), the term $a_{2} / R^{5}$ becomes important for small $R$ and the bubble exhibits oscillatory behavior. The absolute magnitude of the $P_{2}$-shape mode oscillation of this case is relatively smaller, compared to the cases of $a_{20}= \pm 1$. This is because $Q$ is relatively small at the initial stage for a collapsing bubble [see Fig. 3(b)]. However, even in this case, $\left|a_{2} / R\right|$ becomes very large, especially at the final stage of the collapse, and the shape of the bubble is significantly deformed, as shown in Fig. 9(b). It is noteworthy that the bubble evolves from a prolate shape to an oblate shape at the final stage of collapse. This corresponds to the sign change of $a_{2}$ shown in Fig. 8.

There follows a sample problem to demonstrate the effect of the magnetic field on the bubble motion more clearly. Firstly, the growth of an initially spherical bubble in liquid sodium is considered when it enters a low pressure region, such as a venturi tube. For the sake of simplicity, it is assumed that $p_{\infty}$ is abruptly changed and kept constant at $p_{\text {vap }}-p_{\infty}=1$ bar. The properties of liquid sodium at $100^{\circ} \mathrm{C}$ 


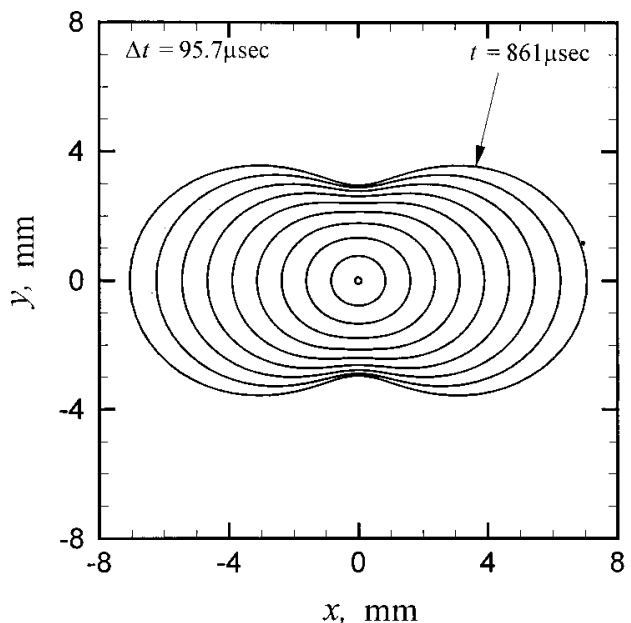

(a)

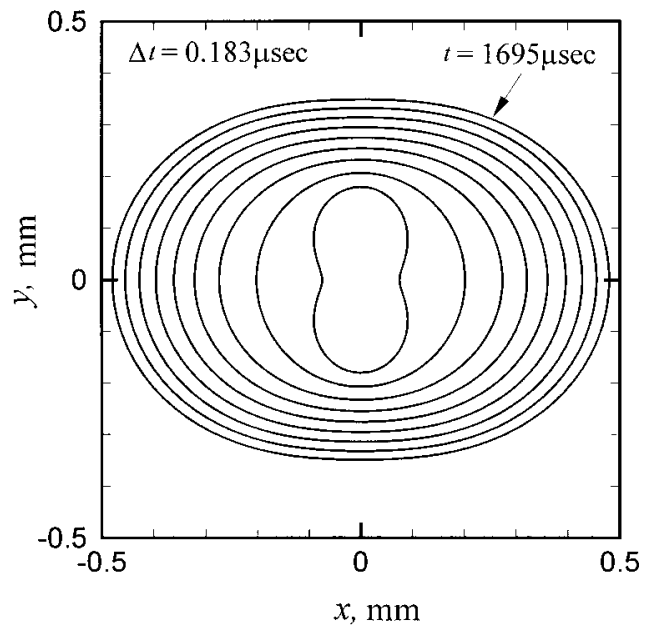

(b)

FIG. 10. The temporal evolution of bubble shape: (a) growing bubble in sodium; (b) collapsing bubble in mercury.

are $\quad \sigma=1.03 \times 10^{7} \Omega^{-1} \mathrm{~m}^{-1}, \quad \rho=928 \mathrm{~kg} / \mathrm{m}^{3}, \quad v=7.39$ $\times 10^{-7} \mathrm{~m}^{2} / \mathrm{s}$, and $\gamma=0.484 \mathrm{~N} / \mathrm{m}$. Let $B_{0}=1$ tesla, and $R_{0}$ $=1.0 \times 10^{-4} \mathrm{~m}$. Then, $N=0.106, R e=1,414$, and $W e$ $=20.9$. Figure 10 (a) shows the temporal evolution of bubble shape. It is shown that the bubble is significantly deformed within a short time. If the bubble grows further, the bubble will become unstable. When $p_{\mathrm{vap}}-p_{\infty}$ becomes smaller, the interaction parameter is increased, and the bubble may be distorted more significantly.

Next, the case of collapse of an initially spherical cavitation bubble in mercury is considered. It is assumed in this case too that $p_{\infty}$ is abruptly increased to some positive values such as the case in which a cavitation bubble leaves the venturi tube. The pressure is assumed to be kept constant so that $p_{\text {vap }}-p_{\infty}=-1$ bar. The properties of mercury at room temperature are $\sigma=10^{6} \Omega^{-1} \mathrm{~m}^{-1}, \rho=13550 \mathrm{~kg} / \mathrm{m}^{3}$, and $\nu$ $=1.15 \times 10^{-7} \mathrm{~m}^{2} / \mathrm{s}$. Let $B_{0}=1$ tesla and $R_{0}=5 \times 10^{-3} \mathrm{~m}$, and then $N=0.135$ and $R e=1.19 \times 10^{5}$. The temporal evolution of bubble shape near the final stage of the collapse process is shown in Fig. 10(b). It is confirmed that the effect of Weber number is, in this case, negligible within the range of $10^{2}-10^{4}$. The overall feature of the shape deformation is similar to that shown in Fig. 9(b), due to the similar interaction parameter and little dependency of cavitation-bubble dynamics on viscosity and surface tension during most of the stage of the collapse process.

\section{COMPARISON WITH THE EXISTING EXPERIMENTAL RESULTS}

It will be meaningful to examine the existing experimental results associated with bubble dynamics in a magnetic field with the results of the present investigation. The existing experimental results to investigate the effect of a magnetic field on cavitation bubble and cavitation erosion are summarized in Table I. As shown in the table, this subject has been a very controversial one. ${ }^{11}$

It should be noted that the interaction parameter, which represents the relative magnitude of the electromagnetic force with respect to the inertia force, is very different in each investigation. In particular, for the results obtained by using tap water, the interaction parameter is too small to influence the behavior of a cavitation bubble within the context of the present investigation. Nevertheless, the experimental result of Shal'nev et al. ${ }^{7}$ and Kamiyama et al. ${ }^{9}$

TABLE I. Summary of previous experimental results concerning the effect of a magnetic field on cavitation bubbles and cavitation erosion.

\begin{tabular}{|c|c|c|c|}
\hline Reference & Working fluid & $N$ & Result \\
\hline $\begin{array}{l}\text { Shalobasov } \\
\text { and Shal'nev (Ref. 6) }\end{array}$ & Tap water & $<10^{-7}$ & $\begin{array}{l}\text { Transverse magnetic field w.r.t. flow } \\
\text { direction significantly increases the } \\
\text { weight loss due to cavitation erosion. }\end{array}$ \\
\hline Shal'nev et al. (Ref. 7) & Tap water & $<10^{-7}$ & $\begin{array}{l}\text { Growth rate of a spark-generated } \\
\text { bubble is increased due to magnetic } \\
\text { field. }\end{array}$ \\
\hline $\begin{array}{l}\text { Kamiyama et al. } \\
\quad \text { (Ref. 9) }\end{array}$ & Tap water & $<10^{-7}$ & $\begin{array}{l}\text { Weight loss due to cavitation erosion } \\
\text { is increased by magnetic field. }\end{array}$ \\
\hline Hammitt et al. (Ref. 11) & Tap water & $<10^{-7}$ & Little effect on cavitation inception. \\
\hline $\begin{array}{l}\text { Perel'man } \\
\text { and Govorskii (Ref. 8) }\end{array}$ & $\begin{array}{l}\text { (Probably) } \\
\text { Sodium }^{11}\end{array}$ & $<0.1$ & $\begin{array}{l}\text { Weight loss due to cavitation erosion } \\
\text { is significantly reduced by magnetic } \\
\text { field. }\end{array}$ \\
\hline $\begin{array}{l}\text { Kamiyama } \\
\text { and Yamasaki (Ref. 10) }\end{array}$ & Mercury & $<0.1$ & $\begin{array}{l}\text { Cavitation inception can be much } \\
\text { delayed by transverse magnetic field } \\
\text { w.r.t. flow direction. }\end{array}$ \\
\hline
\end{tabular}


showed that the behavior of the bubble is significantly influenced by the magnetic field of $N<10^{-7}$. Moreover, the results of Shal'nev et al. ${ }^{7}$ are evidently opposed to the wellknown fact that the magnetic field usually tends to suppress the motion of conducting fluids, which is consistent with the result of the present investigation.

This suggests that there may exist an unknown mechanism controlling the bubble behavior in an effectively dielectric liquid such as tap water in a magnetic field. For example, the Coulomb force exerted on each ion on the bubble surface may change the behavior of a bubble to a certain degree, as discussed by Shalobasov and Shal'nev. ${ }^{6}$ It needs further investigation, considering the influence of charges existing on the bubble surface, to obtain a reliable explanation for such anomalous behavior of a bubble in nonconducting liquids.

On the other hand, the results of Hammitt et al. ${ }^{1,11}$ are seemingly contradictory to the result of Shal'nev et al. ${ }^{7}$ and Kamiyama et al. ${ }^{9}$ However, the influence of a magnetic field, through the results of either the present investigation or Shalobasov and Shal'nev, ${ }^{6}$ is basically driven by the motion of the bubble. As shown in (48) and (49), the influence of a magnetic field on bubble motion is proportional to the radial velocity of the bubble wall. Initially, the radial velocity of a growing bubble is very small, so that the magnitude of the electromagnetic force becomes insufficiently small to suppress the bubble growth. Thus, the magnetic field may have little effect during the initial growing stage of the bubble, unless the magnetic field is overwhelmingly strong. In the case of nuclei induced cavitation, this may contribute to the reason why Hammitt et al. ${ }^{1,11}$ could not find any visible effect of a magnetic field on cavitation inception.

The result of Kamiyama and Yamasaki ${ }^{10}$ of delayed cavitation inception by a magnetic field in a mercury flow is interesting. As discussed above, a weak magnetic field may have little influence on cavitation inception. The delay of inception in this case is conjectured to be due to the increase in static pressure at the venturi tube. The static pressure can be increased by the retarding flow velocity by the Lorentz force directed against the oncoming flow, owing to the magnetic field applied transversely to the flow.

To check the result of Perel'man and Govorskii, ${ }^{8}$ the volume mode of motion of a bubble in the case of ultrasonic cavitation of liquid sodium is calculated by using (48). In the calculation, the interaction parameter was chosen to be 0.1 , which is a little greater than the value used by Perel'man and Govorskii in their experiment. According to the calculated results, the volume of the bubble is hardly influenced by the magnetic field except near the final collapsing stage. This should not be construed as there being no effect of a magnetic field on a growing bubble. One possible reason for such a result is that much greater exciting pressure generated by ultrasonic vibrator than the pressure induced by the Lorentz force dominates the whole growth process of bubbles. ${ }^{1}$

It is anticipated that a magnetic field should be more influential during the collapse phase rather than the growth phase, since the collapsing speed is significantly greater than the growing speed. The present results show that a uniform magnetic field exerts a damping force on volume mode of motion by the dissipation of the kinetic energy of a cavitation bubble. Additionally, the magnetic field destabilizes the collapsing bubble, as shown in Fig. 9(b). A bubble can eventually be disintegrated during the collapse phase. This may reduce the peak amplitude of impulse force on a solid surface generated by the rupture of a bubble attached to the surface. This will certainly reduce the erosion of the metal surface and is consistent with the experimental results of Perel'man and Govorskii.

At the final stage of bubble collapse near a solid boundary, a jet flow is usually formed towards the boundary. The erosion of a metal surface by cavitation bubbles is mainly due to the impact force of the jet flow towards the metal surface. Magnetic fields in general exert a damping force against the flow in a conducting fluid. The magnitude of the damping force is proportional to the cross product of the magnetic flux vector and velocity vector. Then, the strength of the impulsive force due to the jet flow will certainly be reduced. ${ }^{23}$ This fact also supports the results of Perel'man and Govorskii of reduced weight loss in a magnetic field. This could become an additional benefit of using a magnetic field to reduce the damage of metal surfaces caused by cavitation bubbles.

\section{CONCLUSIONS}

The governing equations for the volume and shape modes of oscillation are derived for a bubble under a uniform magnetic field. It is shown that the magnetic-field exerts a damping force on the volume mode. Thus, both the growing and collapsing speeds in volume mode are reduced by the magnetic field. The magnetic field also affects the $P_{2}$-mode motion of the bubble wall by contributing to a forcing term. Within the validity of the present linearized solution for the shape mode, the $P_{2}$ - mode of an either growing or collapsing bubble becomes unstable by the magnetic field, regardless of the initial conditions.

\section{ACKNOWLEDGMENTS}

The authors wish to acknowledge the funding support of the Korea Science and Engineering Foundation and the Advanced Fluids Engineering Research Center of the Pohang University of Science and Technology.

${ }^{1}$ F. G. Hammitt, Cavitation and Multiphase Flow Phenomena (McGrawHill, New York, 1980).

${ }^{2}$ D. L. Frost, "Dynamics of explosive boiling of a droplet," Phys. Fluids 31, 2554 (1988).

${ }^{3}$ P. Selvaraj, K. N. Seetharamu, and G. Vaidyanathan, "Large leak sodiumwater reaction analysis of an LMFBR steam generator using a variable temperature spherical bubble model," Nucl. Eng. Des. 123, 87 (1990).

${ }^{4}$ R. Jonas, W. Schütz, and A. Reetz, "Model calculations and experimental verification of the bubble behavior in a Bethe-Tait accident," Nucl. Eng. Des. 118, 71 (1990).

${ }^{5}$ S. Lesin, A. Baron, I. Zilberman, H. Branover, and J. C. Merchuk, "Direct contact boiling studies applicable for liquid metal MHD systems," Proceedings of the Second International Conference on Multiphase Flow, Kyoto, Japan, PC2-1 (1995).

${ }^{6}$ I. A. Shalobasov and K. K. Shal'nev, "Effect of an external magnetic field on cavitation and erosion damage," Heat Transfer-Sov. Res. 3, 141 (1971).

${ }^{7}$ K. K. Shal'nev, I. A. Shalobasov, S. P. Kozirev, and E. V. Haldeev, "Cavitation bubble in magnetic field," Proceedings of the Conference on Fluid Machinery, Hungary, 1021 (1975).

${ }^{8}$ R. G. Perel'man and E. V. Govorskii, "Effect of a constant magnetic field 
on cavitation erosion in an electrically conducting liquid," Sov. Phys. Dokl. 16, 164 (1971).

${ }^{9}$ S. Kamiyama, K. Koike, and H. Kawaguchi, "Effects of magnetic field on cavitation damage," Proceedings of the Second International Conference on Cavitation, 51 (1983).

${ }^{10} \mathrm{~S}$. Kamiyama and T. Yamasaki, "Cavitation in a flow of mercury in a transverse magnetic field," Magnetohydrodynamics 10, 277 (1974).

${ }^{11}$ F. G. Hammitt, E. Yilmaz, O. Ahmed, N. R. Bhatt, and J. Mikielewicz, "Effects of magnetic and electric fields upon cavitation in conducting and non-conducting liquids," Cavitation and Polyphase Flow Forum, ASME, 26 (1975).

${ }^{12}$ C. P. C. Wong, G. C. Vliet, and P. S. Schmidt, "Magnetic field effects on bubble growth in boiling liquid metals," Proceedings of the Second Topical Conference on Technology of Nuclear Fusion 2, 407 (1976).

${ }^{13} \mathrm{C}$. Vivès, "Effects of forced electromagnetic vibrations during the solidification of aluminum alloys: Part I. Solidification in the presence of crossed alternating electric fields and stationary magnetic fields," Metall. Mater. Trans. B 27, 445 (1996).

${ }^{14}$ R. C. Weast, S. M. Selby, and C. D. Hodgman, Handbook of Chemistry and Physics, 45th ed. (The Chemical Rubber Co., Cleveland, OH, 1964).
${ }^{15}$ A. Vogel, W. Lauterborn, and R. Timm, "Optical and acoustic investigations of the dynamics of laser-produced cavitation bubbles near a solid boundary," J. Fluid Mech. 206, 299 (1989).

${ }^{16}$ R. Moreau, Magnetohydrodynamics (Kluwer Academic, Dordrecht, 1990).

${ }^{17}$ G. K. Batchelor, An Introduction to Fluid Dynamics (Cambridge University Press, London, 1967).

${ }^{18}$ A. Prosperetti, "Viscous effects on perturbed spherical flows," Q. Appl. Math. 34, 339 (1977)

${ }^{19}$ G. Arfken, Mathematical Methods for Physicists, 3rd ed. (Academic, Orlando, 1985).

${ }^{20}$ I. S. Kang and L. G. Leal, "Small-amplitude perturbations of shape for a nearly spherical bubble in an inviscid straining flow (steady shapes and oscillatory motion)," J. Fluid Mech. 187, 231 (1988).

${ }^{21}$ A. Prosperetti and G. Seminara, "Linear stability of a growing or collapsing bubble in a slightly viscous liquid," Phys. Fluids 21, 1465 (1978).

${ }^{22}$ R. B. Chapman and M. S. Plesset, "Nonlinear effects in the collapse of a nearly spherical cavity in a liquid," J. Basic Eng. 94, 142 (1972).

${ }^{23}$ P. A. Davidson, "Magnetic damping of jets and vortices," J. Fluid Mech. 299, 153 (1995). 